\title{
Vitamin D: The Immunologic Role and its Effect on Human Pathophysiology
}

\author{
${ }^{1}$ William Whalen, ${ }^{2}$ Edward Werner Cook and ${ }^{1}$ Nitya G. Chakraborty \\ ${ }^{I}$ Department of Medicine, University of Connecticut Health Center, Farmington CT 06032, USA \\ ${ }^{2}$ Department of Science and Math, Tunxis Community College, Farmington, CT 06032, USA
}

Article history

Received: 31-12-2014

Revised: 19-03-2015

Accepted: 19-03-2015

Corresponding author:

Nitya G. Chakraborty

Department of Medicine,

University of Connecticut Health

Center, Farmington CT 06032,

USA

Email: chakraborty@uchc.edu

\begin{abstract}
There are several reasons why understanding the role of vitamin $\mathrm{D}$ in the immune system is worthy of widespread attention. Our knowledge of the immune system is crucial for understanding human health and disease. New ideas for vitamin D and life style in the twenty-first century build directly on our understanding of immunology-from the development of new immune-based cancer drugs to advancing treatments for common diseases, such as autoimmunity and allergies. The scientists and physicians must be inspired to tackle the vast array of unmet immunological needs and the application of old theories-the effectiveness of vaccines, for example-can benefit largely from our understanding of Vitamin D and its role in immunology. The role of immune system is to seek out and destroy dangerous bacteria, viruses, fungi and uncontrolled tumor (cancer) growth. Its activities connect with other body systems and influences, our metabolism and hormone levels and controls how well we feel. Nutrition and our mental health are all connected to our ability to fight infections and abnormal cell growth. In this article we will mainly focus on Vitamin D and its immunological effects on pulmonary disease, tuberculosis and cancer.
\end{abstract}

Keywords: Vitamin D, Immune System, Pathophysiology

\section{Introduction}

Sunny D, the Sunshine Vitamin D, a lipophilic secosteroid, plays a vital role in Calcium (Ca) homeostasis, immune cells, especially T-cell activation, pulmonary physiology and pathophysiology such as Asthma, Tuberculosis and COPD. Historically, Vitamin $\mathrm{D}$ was found to be the agent preventing Rickets (bow legs) in children and facilitated transport of $\mathrm{Ca}$ to bone. The precursor of active Vitamin D, 7dehydrocholesterol, undergoes a series of biosynthetic reactions resulting in the active form of Vitamin D, calcitriol. Calcitriol mediates its effects through a nuclear Vitamin D Receptor (VDR), which subsequently binds to DNA, leading to downstream effects. In respect to classical T-Cell activation, the VDR-1, $25(\mathrm{OH})_{2} \mathrm{D}_{3}$ complex translocates to the nucleus causing PLC- $\gamma 1$ gene activation and increased protein expression, which is responsible for greater T-Cell Receptor (TCR) responsiveness. It has been shown that VDR gene methylation is varied across different ethnic groups. Additionally, vitamin D plays a role on pulmonary function, as higher Forced Expiratory Volumes after one second $\left(\mathrm{FEV}_{1}\right)$ is correlated with increased amounts of 25-hydroxy vitamin D within the serum. Due to the role that Vitamin D plays in normal physiology and immunology, one can suspect that it can play a role in immune mediated pulmonary pathophysiology. Vitamin D insufficiency at baseline results in increased odds of having a severe asthma exacerbation, increased severity of emphysema and tuberculosis status. There also exists a relationship between inflammatory markers of Chronic Obstructive Pulmonary Disease (COPD) and bronchodilator reversibility, with increased levels of Vitamin D resulting in decreased inflammatory markers and increased bronchodilator reversibility. This review will provide a brief overview of the biosynthesis of Vitamin D and will discuss the role that Vitamin D and the Vitamin D Receptor (VDR) in T-Cell activation, asthma, COPD, tuberculosis and metastatic cancer.

\section{Overview of the Vitamin D Biosynthesis and Interaction with Vitamin D Receptor}

The initial building block of Vitamin D is 7dehydrocholesterol, Pre- $\mathrm{D}_{3}$, which is converted to 
Vitamin $\mathrm{D}_{3}$ (cholecalciferol) upon ultraviolet $\mathrm{B}$ radiation (wavelength 290-315 $\mathrm{nm}$ ) exposure. The majority of Vitamin $D_{3}$ is obtained through this photo activation pathway; therefore individuals who live above 35 degrees latitude are at increased risk for Vitamin D deficiency without dietary supplementation (Holick and Michael, 2007). Vitamin $\mathrm{D}_{3}$ and Vitamin $\mathrm{D}_{2}$ (ergocalciferol), can also be absorbed through the gut from dietary sources such as milk, fish, fortified foods and Vitamin $\mathrm{D}_{2}$ supplements. When it enters the circulation it binds to vitamin D-binding protein in the bloodstream and is transported to the liver.

Vitamin $\mathrm{D}_{3}$ is metabolized in the liver by the enzyme 25-hydroxylase, encoded by the gene CYP271A1, to 25Hydroxycholecalciferol (Calcidiol). Calcidiol, an inactive precursor, is transported to the proximal tubules of the kidney where it is converted into the active metabolite of 1,25-dihydroxyvitamin $\mathrm{D}\left[1,25(\mathrm{OH})_{2} \mathrm{D}\right]$ via the enzyme 25-hydroxyvitamin D-1 $\alpha$-hydroxylase (1 $\alpha$-hydroxylase), encoded by the gene CYP27B1 (Fig. $1)$. Regulation of $1 \alpha$-hydroxylase is under tight control with levels of calcium, phosphorus, fibroblast growth factor 23 and Parathyroid Hormone (PTH) all playing a role in regulation. $1,25(\mathrm{OH})_{2} \mathrm{D}$ decreases production of itself through negative feedback on $1 \alpha$-hydroxylase. Additionally $1,25(\mathrm{OH})_{2} \mathrm{D}$ imparts negative feedback on PTH secretion. There exists enzymatic counter regulatory processes as well, such as 25-hydroxyvitamin $\mathrm{D}$-24-hydroxylase (24-OHase), which is activated by $1,25(\mathrm{OH})_{2} \mathrm{D}$. 24-OHase acts on $1,25(\mathrm{OH})_{2} \mathrm{D}$ converting it to the metabolically inert calcitroic acid, which is then excreted in the bile (Fig. 1). This negative feedback loop prevents auto toxicity from photosynthesis and equilibrium can be achieved within a half-hour with individuals responsive to photosynthesis. Calcidiol is the major circulating metabolite and is what is measured when determining deficiency or intoxication of Vitamin D. A patient is labeled deficient if circulating levels of Calcidiol are less than $20 \mathrm{ng} \mathrm{mL}^{-1}$ and intoxicated when levels exceed $150 \mathrm{ng} \mathrm{mL}^{-1}$ (Holick and Michael, 2007).

\section{Vitamin D and T-Cell Activation}

$\mathrm{T}$ lymphocytes ( $\mathrm{T}$ Cells) are the major lymphocytes of cell-mediated immunity, while B lymphocytes (B Cells) represent the major component of humoral immunity. Both cell lines mediate their response to antigen through different mechanisms with the T cell and $B$ cell using the T Cell antigen Receptor (TCR) and the $B$ cell antigen receptor respectively. These antigen receptors differ in that the $\mathrm{B}$ cell antigen receptor can undergo affinity maturation to make a stronger bond to a specific antigen while the $\mathrm{T}$ cell receptor is limited, in that it must undergo a process termed 'functional avidity maturation.' $\mathrm{T}$ cells exist predominantly in two states:
Naïve and activated, differing in that the latter has encountered its cognate antigen thus activating the $\mathrm{T}$ cell, leading to greater $\mathrm{T}$ cell proliferation and cytokine production than naïve $\mathrm{T}$ cells.

The TCR signaling model is as follows: Ligation of the TCR induces and activates Lck, a tyrosine kinase. The next step is phosphorylation of the $\mathrm{CD}_{3}$ co-receptor complex and $\zeta$-chains of the TCR. Associated with the $\zeta$ chains, the protein Zap70, becomes activated and then phosphorylates the membrane adaptor Lat, which consequently recruits and activates PLC- $\gamma 1$ an Src homology containing protein. Activated PLC- $\gamma 1$ goes on to hydrolyze phophatidylionositol-4,5-bisphosphate $\left(\mathrm{PIP}_{2}\right)$ to inositol-3,4,5-triphosphate $\left(\mathrm{IP}_{3}\right)$, which induces intracellular calcium release and Diacylglycerol (DAG), which controls protein kinase C and RasGRP (activator of the GTPase RAS). Activation of the RAS system will then induce Mitogen-Activated Protein Kinase (MAPK), leading to production of transcription factors, gene expression and finally entry into the cell cycle (Fig. 2).

As previously discussed and illustrated in Fig. 2, PLC- $\gamma 1$ is crucial intermediate that can also control MAPK cascade. An alternative TCR signaling pathway, other than the one previously described, that leads to activation of MAPK p38. After zap 70 is activated, it will directly phosphorylate p38 leading to its activation, effectively bypassingLat and PLC- $\gamma 1$ (von Essen et al., 2010).

Vitamin D Receptor (VDR) and vitamin D play an intimate role in the functioning of the TCR. Naïve T cells initially have very low expression of PLC- $\gamma 1$ and VDR, correlating with low TCR responsiveness. When TCR signaling occurs via the alternate pathway, induction of VDR can be achieved. This will bind to its ligand $1,25(\mathrm{OH})_{2} \mathrm{D}_{3}$, translocate to the nucleus and promote the transcription and translation of the PLC- $\gamma 1$ gene. Therefore initial TCR signaling via the p38 alternate pathway can lead to successive induction of VDR and PLC- $\gamma 1$, requirements that must be met for activation through the classical pathway.

\section{Role of Vitamin D in Pulmonary Function}

Pulmonary function is clinically measured through spirometry, lung volumes, diffusion capacity across the respiratory epithelium and through the use of arterial blood gasses (Robbins, 2010). Spirometry allows a clinician to calculate the Forced Expiratory Volume after one second (FEV1), Forced Vital Capacity (FVC; amount of air that can be forcibly blown out after full inspiration) and the FEV1/FVC ratio. FEV1 values range between $80-120 \%$ of normalized values and the FEV1/FVC ratio should be approximately $75-80 \%$ in healthy adults. These values vary in respect to cigarette smoke exposure, environmental exposures, diet, 
exercise, age and ethnicity, to name a few. Disease pathology also alters these values as FEV1/FVC ratio is reduced $(<80 \%)$ in obstructive pulmonary disease (IE
Asthma and COPD) while FEV1/FVC ratio is unchanged or increased in restrictive lung disease (IE obesity and pulmonary fibrosis) (Robbins, 2010).

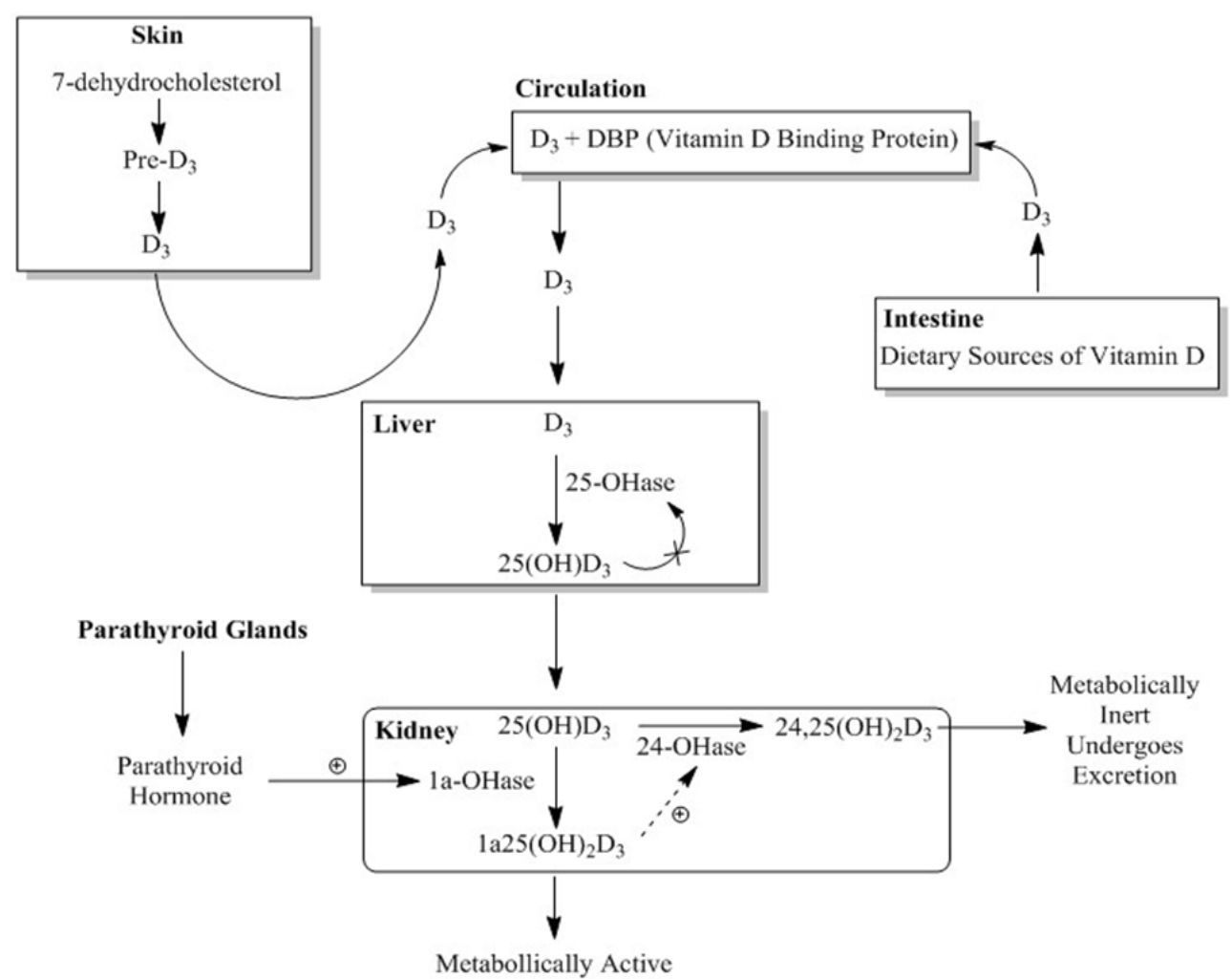

Fig. 1. Biosynthesis of Vitamin D: The pathways of synthesis of Vitamin D from the precursor its utilization and excretion in from the body

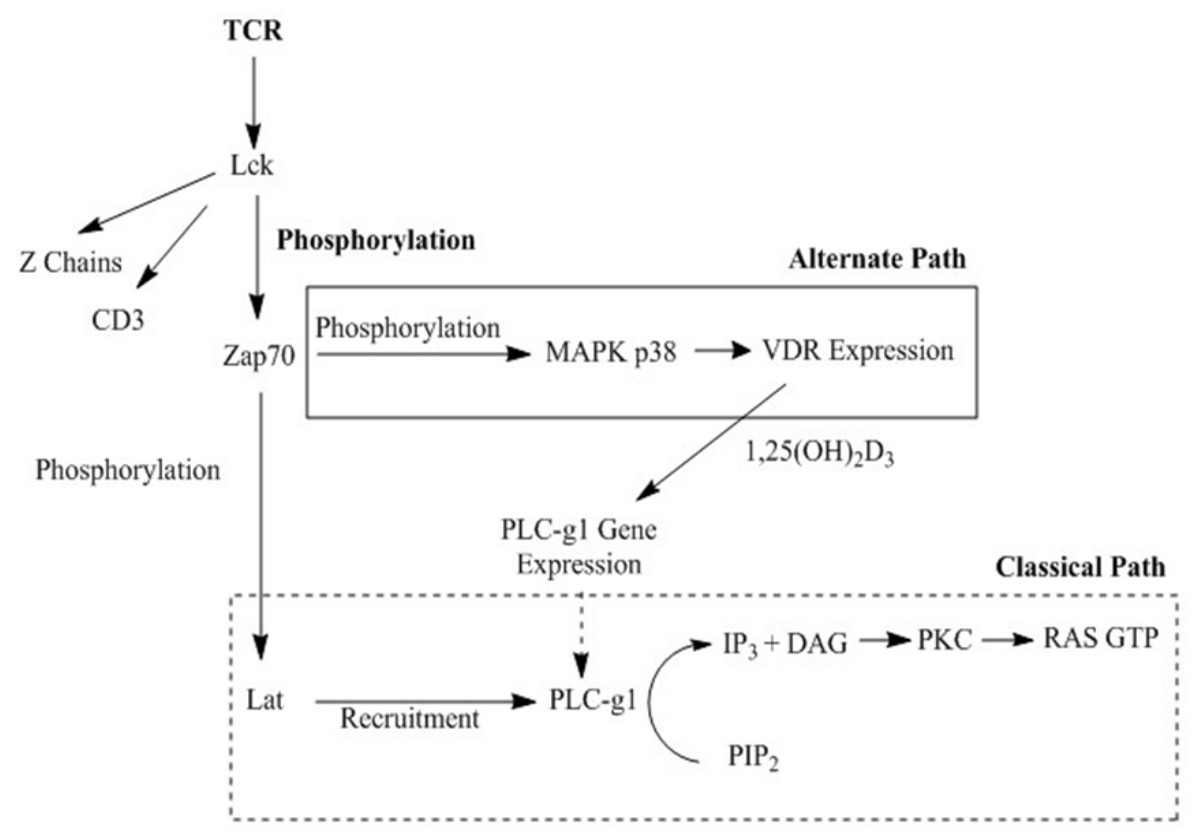

Fig. 2. T cell receptor activation: Role of the isozyme phospholipase $\mathrm{C} \gamma-1$ and activation/ expression of T cell receptor 
Due to the roles that vitamin D plays in bone development and maintenance, the cardiovascular system and in the immune system, it is reasonable to consider that Vitamin D affects pulmonary function as well. Black and Scragg (2005) investigated the relationship between serum 25-Hydroxyvitamin D and pulmonary function. The analysis was a cross-sectional survey of the US civilian non-institutionalized population (NHANES III) from 1988 to 1994. The data from the study suggests that there is a dose-response relationship between serum concentration of vitamin D and FEV1 after the adjustment for age, gender, ethnicity, height and smoking; all of which can affect lung function. FEV1 was $126 \mathrm{~mL}$ and the mean FVC was $172 \mathrm{~mL}$ greater in the highest quintile $\left(>85.7 \mathrm{nmol} \mathrm{L}{ }^{-1}\right.$ Vitamin D) compared with the lowest quintile $(<40.4$ nmol L ${ }^{-1}$ Vitamin D; $\left.<<0.0001\right)$. Furthermore this study adjusted for physical activity, intake of Vitamin D and supplements along with other serum antioxidants and still found a difference between the highest and lowest quintile, with the difference for FEV1 being $106 \mathrm{~mL}$ and FVC being $142 \mathrm{~mL}(\mathrm{p}<0.0001)$. There was no difference in the FEV1/FVC ratio between the highest and lowest quintiles, indicating that the clinical picture of vitamin D deficiency is similar to that of restrictive lung pathology. The authors of the paper theorize that vitamin D influences pulmonary function through tissue remodeling and repair, which is ongoing throughout life. Vitamin D can influence tissue remodeling through the inhibition of matrix metalloproteinases and through its effects on fibroblast proliferation (Black and Scragg, 2005).

\section{Role of Vitamin D in Asthma}

Asthma a common pulmonary disorder affecting 18.7 million adults and 7 million children within the United States alone. In accordance with the United States Center of Disease Control, asthma resulted in over \$56 billion dollars in healthcare related costs and in more than 400,000 hospitalizations. Asthma is characterized by inflammation and narrowing of the airways secondary to increased mucous production and increased contractility of the smooth muscle cells that surround the airway. An asthma exacerbation can be caused by irritants in the air, respiratory infections and exercise which, leads to increased swelling and bronchospasm of the airways (Bozzetto et al., 2012; Brehm et al., 2010).

It has been classically thought that the Kidney activates 25-OH-Vitamin $\mathrm{D}_{3}$ to the active form of 1,25$\mathrm{OH}-$ Vitamin $\mathrm{D}_{3}$ through the enzyme 1ahydroxylase, however this enzyme is found in abundance within airway epithelial cells, allowing for conversion to occur within the pulmonary system. This allows for vitamin D to become localized within the respiratory epithelium, where it has been shown to induce antimicrobial proteins such as cathelicidin and defensin $\beta 4$ along with antiinflammatory cytokines therefore mitigating the extent of the asthma exacerbation (Brehm et al., 2010).

In a recent study examining serum vitamin D levels and severe asthma exacerbations in children, with mild to moderate persistent asthma, were correlated to the number of hospitalizations or emergency department visits. It was found that children who were considered Vitamin D deficient $\left(<30 \mathrm{ng} \mathrm{mL}{ }^{-1}\right)$ had increased odds of going to the emergency department or being hospitalized due to an asthma exacerbation. Concordantly children with Vitamin D deficiency had a slightly decreased $\mathrm{FEV}_{1}$ in comparison to those with adequate Vitamin D levels. Utilizing the mechanism of Vitamin D outlined in the previous paragraph, Vitamin D most likely works through anti-inflammatory and anti-microbial mechanisms therefore allowing the host to handle upper respiratory tract infections more efficiently and diminishing the inflammatory response (Bozzetto et al., 2012), which triggers the asthma exacerbation.

\section{Vitamin D and Chronic Obstructive Pulmonary Disease}

Chronic Obstructive Pulmonary Disease (COPD), defined by the world health organization as a "Common preventable and treatable disease, is characterized by airflow limitation that is usually progressive and associated with an enhanced chronic inflammatory response in the airways and the lung to noxious particles or gasses. Exacerbation and comorbidities contribute to the overall severity in individual patients." COPD (2014a). COPD is associated with high morbidity and was responsible for 40 deaths per 100,000 individuals in 2010 within the United States alone (COPD, 2014b). Disease subtypes of COPD include emphysema, chronic bronchitis and chronic obstructive asthma. COPD carries a large healthcare burden due to the frequent office visits, hospitalizations due to exacerbations and the need for chronic therapy (Pauwels et al., 2001).

The association of Vitamin D with pulmonary and immune function was previously mentioned, therefore one may suspect that if Vitamin D plays a role in normal physiology, there is a role for Vitamin $\mathrm{D}$ in a pathological state. The hallmark of COPD on pulmonary function is measured clinically with the use of a spirometer through measurement of $\mathrm{FEV}_{1}$ and $\mathrm{FVC}$ and the ratio of the former to the latter. COPD causes a reduction in both $\mathrm{FEV}_{1}$ and $\mathrm{FVC}$ and a reduction in the ratio of $\mathrm{FEV} 1 / \mathrm{FVC}$, with an $\mathrm{FEV}_{1} / \mathrm{FVC}$ of 0.7 being considered diagnostic. Clinically, COPD can be assessed with a patient's response to bronchodilator treatment; the 
6 min walk test and the St. George Respiratory Questionnaire score. The pathology of COPD includes an increased number of goblet cells, mucus gland hyperplasia, fibrosis, narrowing and reduction in the number of small airways leading to airway collapse. Subsequently the lung parenchyma undergoes change as well with destruction to the acinus (respiratory bronchiole, alveolar ducts, alveolar sacs and alveoli). The pathological process in emphysema, chronic bronchitis and asthma is driven through T-Cells and cytokines (Robbins, 2010).

There exists a strong relationship between emphysema and 25(OH)D levels. This relationship was perceived clinically as Vitamin D levels correlated with improved six minute walk time and bronchodilator response. The pathology of emphysema is complex, but vitamin $\mathrm{D}$ may play a role in modulating the immune cells that release elastases and cytokines that induce acini destruction, through inhibition of matrix metalloprotienases or, as the authors of the cited paper speculate, though pulmonary structure remodeling (Berg et al., 2013).

\section{Role of Vitamin D in Tuberculosis}

Tuberculosis (TB) is caused by the bacterium Mycobacterium Tuberculosis (MTB), with the lungs being the main site of infection. TB can be manifested in two forms, active $\mathrm{TB}$ and latent $\mathrm{TB}$, the presentation can be varied therefore it is crucial to take a comprehensive history and have low clinical threshold to, when warranted, start the work up. In regards to pulmonary symptoms and pathology TB can lead to complications such as hemoptysis, pneumothorax, bronchiectasis, extensive pulmonary destruction, malignancy and chronic pulmonary aspergillosis. Treatment for TB broadly revolves around four drugs: Isoniazid, rifampin, pyrazinamide and ethambutol. The response to TB is monitored by repeat sputum smear two months after initiation of therapy to see if MTB is still present. The pathophysiology and treatment of TB will not be discussed, as it is outside the scope of the review (Zumla et al., 2013).

As discussed previously, Vitamin D interacts with the VDR located on all immune cells within the body. VDR is increased in the presence of $\mathrm{TB}$, secondary to TB antigenic stimulation of macrophage and monocytic toll like receptors, inducing cathelicidin (part of the antimicrobial polypeptide family) mediated killing. Vitamin D also plays a role in the production of Interferon $\gamma$ (IFN- $\gamma$ ), which is an essential pro-inflammatory cytokine that helps in the resistance to TB infection as it induces granuloma formation.

The SUCCINCT study (Salahuddin et al., 2013) investigated the effect of therapeutic levels of two doses of Vitamin D (600,000 IU) given at the start and one month after the initiation of TB therapy. It was found that only individuals who were deemed to have deficient serum Vitamin D concentrations $\left(<20 \mathrm{ng} \mathrm{mL}^{-1}\right)$ had increased levels of IFN- $\gamma$ secretion. This may suggest that there exists a threshold of serum Vitamin $\mathrm{D}$ needed to produce adequate pro-inflammatory cytokine levels for the containment of TB. Additionally, all individuals whom received vitamin D supplementation experienced weight gain and had less residual disease by chest radiograph; decreased zone involvement throughout the lung fields and reduction in cavity size by fifty percent or more. The author's note two limitations to the study; one that they did not follow patients out to the six month mark which is the end term of the treatment and they did not monitor dietary intake of vitamin $\mathrm{D}$ from the participants of the study.

\section{Role of Vitamin D in Incidences of Cancer}

Serum concentrations of Vitamin D differ among the races. Residents of the northeastern United States and individuals with more skin pigmentation have an increased risk of deficiency (Grant and Garland, 2014). A database search yielded 63 studies of vitamin D status in relation to several types of cancer risk, including colon, breast, prostate and ovarian cancer. Several studies were done that assessed the association of vitamin D receptor genotype with cancer risk (Grant and Garland, 2014). The majority of studies found a protective relationship between sufficient vitamin D status and lower risk of cancer. The evidence suggests that efforts to improve vitamin D status, for example by vitamin D supplementation, could reduce cancer incidence and mortality at low cost, with few or no adverse effects (Grant and Garland, 2014).

Induction of Phospholipase C (PLC- $\gamma 1$ ), one of the isozymes, an important signaling protein downstream of many extra-cellular stimuli was found to be dependent on vitamin $\mathrm{D}$ and expression of the Vitamin D Receptor (VDR) (Reddy, 2013). Vitamin D deficiency has been related to metastatic breast, prostate and colon cancer, which could be associated with improper coordination between different subsets of $\mathrm{T}$ lymphocytes like regulatory (Treg) or Cytotoxic $\mathrm{T}$ Lymphocytes (CTL). It is not known how circulating $\mathrm{T}$ cells are different in different races of people in terms of downstream signaling after the extra cellular stimulations. It has been shown that people of African-American community (AA) are at nearly twice the risk of metastatic cancers and death as compared with white people, which may be due to increased Vitamin D deficiency amongst individuals within the AA community. Metastatic diseases remain 
a serious challenge in cancer therapy. It has been postulated that metastatic cancer might be the result of failure of cytotoxic T lymphocyte (mainly CD8) responses to control the abnormal cell growth and/or due to the presence of over active regulatory $\mathrm{T}$ lymphocyte responses (mainly CD4), that block the CTL activity.

Vitamin D and its metabolites reduce the incidence of many types of cancer by inhibiting tumor angiogenesis, (Iseki et al., 1999; Majewski et al., 1996; Shokravi et al., 1995; Mantell et al., 2000) stimulating mutual adherence of cells, (Palmer et al., 2001) and enhancing intercellular communication through gap junctions, (Fujioka et al., 2000) thereby strengthening the inhibition of proliferation that results from tight physical contact with adjacent cells within a tissue (contact inhibition). Vitamin D metabolites help maintain a normal calcium gradient in the colon epithelial crypts, (Palmer et al., 2001) and high serum levels of $25(\mathrm{OH}) \mathrm{D}$ are associated with markedly decreased proliferation of noncancerous but high-risk epithelial cells in the colon (Lipkin and Newmark, 1985; Holt et al., 2002). 1,25(OH $)_{2} \mathrm{D}$ inhibits mitosis of breast epithelial cells (Campbell et al., 1997). Pulsatile release of ionized calcium from intracellular stores, including the endoplasmic reticulum, induces terminal differentiation and apoptosis, (Mathiasen et al., 2002) and $1,25(\mathrm{OH})_{2} \mathrm{D}$ enhances this release.

\section{Conclusion}

Vitamin D is lipophilic vitamin that is tightly regulated through a complex biochemical pathway within the body. The photosynthesis of Vitamin D in the skin and subsequent complex effects in regions remote show that this biomolecule behaves as a hormone and that the name is essentially historical. The effects activated Vitamin D induces on the body are far reaching and include its effects on the immune system along with pulmonary function and pulmonary pathophysiology. Keeping the extensive effects of Vitamin $\mathrm{D}$ in mind and the low cost of supplementation, clinicians should consider screening for deficiency and treat when appropriate.

\section{Acknowledgement}

This work was supported by a grant from Department of Medicine and Neag comprehensive Cancer Center, University of Connecticut Health Center, Farmington, Connecticut.

\section{Disclaimer}

Authors do not have any financial interest for this article.

\section{Author's Contributions}

William Whalen: Planning, researching, organizing, writing and partial editing.

Edward Werner Cook: Planning, researching, organizing and partial editing.

Nitya G. Chakraborty: Planning, researching, organizing, writing and partial editing.

\section{Ethics}

This review article is original and contains unpublished material. The corresponding author confirms that all of the other authors have read and approved the manuscript and no ethical issues involved.

\section{References}

Berg, I., C. Hanson, H. Sayles, D. Romberger and A. Nelson et al., 2013. Vitamin D, vitamin D binding protein, lung function and structure in COPD. Respir. Med., 107: 1578-1588. DOI: $10.1016 /$ j.rmed.2013.05.010

Black, P.N. and R. Scragg, 2005. Relationship between serum 25-hydroxyvitamin $\mathrm{D}$ and pulmonary function in the third national health and nutrition examination survey. Chest, 126: 3792-3798. DOI: $10.1378 /$ chest.128.6.3792

Bozzetto, S., S. Carraro, G. Giordano, A. Boner and E. Baraldi, 2012. Asthma, allergy and respiratory infections: The vitamin D hypothesis. Allergy, 67: 10-17. DOI: 10.1111/j.1398-9995.2011.02711.x

Brehm, J.M., B. Schuemann, A.L. Fuhlibrigge, B.W. Hollis and R.C. Strunk et al., 2010. Serum vitamin $\mathrm{D}$ levels and severe asthma exacerbations in the childhood asthma management program study. J. Allergy Clin. Immunol., 126: 52-58. DOI: 10.1016/j.jaci.2010.03.043, PMID: 20538327

Campbell, M.J., G.S. Reddy and H.P. Koeffler, 1997. Vitamin $\mathrm{D}_{3}$ analogs and their 24-oxo metabolites equally inhibit clonal proliferation of a variety of cancer cells but have differing molecular effects. J. Cell Biochem., 66: 413-425. PMID: 9257197

COPD, 2014a. CDC data and statistics. Chronic Obstructive Pulmonary Disease.

COPD, 2014b. COPD data, statistics and surveillance. Chronic Obstructive Pulmonary Disease.

Fujioka, T., Y. Suzuki, T. Okamoto, N. Mastushita and M. Hasegawa et al., 2000. Prevention of renal cell carcinoma by active vitamin D3. World J. Surg., 24: 1205-1210. DOI: $10.1007 / \mathrm{s} 002680010206$

Pauwels, R., A. Buist, P. Calverley, C. Jenkins and S. Hurd, 2001. Global strategy for the diagnosis, management and prevention of chronic obstructive pulmonary disease. Am. J. Respir. Critical Care Med., 163: 1256-1276. DOI: 10.1164/ajrccm.163.5.2101039 
Grant, W.B. and C.F. Garland, 2014. Vitamin D has a greater impact on cancer mortality rates than on cancer incidence rates. BMJ, 48: g2862-g2862. DOI: $10.1136 /$ bmj.g2862

Holick, M.F. and M.D. Michael, 2007. Vitamin D deficiency. N Engl. J. Med., 357: 266-281. DOI: 10.1056/NEJMra070553

Holt, P.R., N. Arber, B. Halmos, K. Forde and H. Kissileff et al., 2002. Colonic epithelial cell proliferation decreases with increasing levels of serum 25-hydroxy vitamin D. Cancer Epidemiol. Biomarkers Prev., 11: 113-119. PMID: 11815408

Iseki, K., M. Tatsuta, H. Uehara, H. Iishi and H. Yano et al., 1999. Inhibition of angiogenesis as a mechanism for inhibition by La-hydroxyvitamin D3 and 1,25dihydroxyvitamin D3 of colon carcinogenesis induced by azoxymethane in Wistar rats. Int. J. Cancer, 81: 730-733. DOI: 10.1002/(SICI)10970215(19990531)81:5<730::AID-IJC11>3.0.CO;2-Q

Lipkin, M. and H. Newmark, 1985. Effect of added dietary calcium on colonic epithelial-cell proliferation in subjects at high risk for familial colonic cancer. N Engl. J. Med., 313: 1381-1384. DOI: 10.1056/NEJM198511283132203

Majewski, S., M. Skopinska, M. Marczak, A. Szmurlo and W Bollag et al., 1996. Vitamin D3 is a potent inhibitor of tumor cell-induced angiogenesis. J. Invest. Dermatol Symp. Proc., 1: 97-101. PMID: 9627702

Mantell, D.J., P.E. Owens, N.J. Bundred, E.B. Mawer

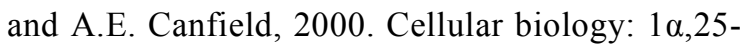
dihydroxyvitamin D3 inhibits angiogenesis in vitro and in vivo. Circ. Res., 87: 214-220. DOI: 10.1161/01.RES.87.3.214

Mathiasen, I.S., I.N. Sergeev, L. Bastholm, F. Elling and A.W. Norman et al., 2002. Calcium and calpain as key mediators of apoptosis-like death induced by vitamin D compounds in breast cancer cells. J. Biol. Chem., 277: 30738-30745. PMID: 12072431
Palmer, H.G., J.M. Gonzalez-Sancho, J. Espada, M.T. Berciano and I. Puig et al., 2001. Vitamin $\mathrm{D}_{3}$ promotes the differentiation of colon carcinoma cells by the induction of E-cadherin and the inhibition of $\beta$-catenin signaling. J. Cell Biol., 154: 369-387. DOI: $10.1083 /$ jcb.200102028

Reddy, K.K., 2013. Vitamin D level and basal cell carcinoma, squamous cell carcinoma and melanoma risk. J. Investigative Dermatol., 133: 589-592. DOI: $10.1038 /$ jid.2012.427

Robbins, S., 2010. Robbins and Cotran Pathologic Basis of Disease. 8th Edn., Saunders/Elsevier, Philadelphia, PA.

Salahuddin, N., F. Ali, Z. Hasan, N. Rao and M. Aqeel et al., 2013. Vitamin D accelerates clinical recovery from tuberculosis: Results of the SUCCINCT study [Supplementary cholecalciferol in recovery from tuberculosis]. A randomized, placebo-controlled, clinical trial of vitamin D supplementation in patients with pulmonary tuberculosis'. BMC Infect. Dis., 13: 22-22. DOI: $10.1186 / 1471-2334-13-22$

Shokravi, M.T., D.M. Marcus, J. Alroy, K. Egan and M.A. Saornil et al., 1995. Vitamin D inhibits angiogenesis in transgenic murine retinoblastoma. Invest. Ophthalmol. Vis. Sci., 36: 83-87.

PMID: 7529753

von Essen, M.R., M. Kongsbak, P. Schjerling, K. Olgaard and N. Odum et al., 2010. Vitamin D controls $\mathrm{T}$ cell antigen receptor signaling and activation of human $\mathrm{T}$ cells. Nat. Immunol., 11: 344-349. DOI: 10.1038/ni.1851

Zumla, A., M. Raviglione, R. Hafner and C.F. von Reyn, 2013. Tuberculosis. N Engl. J. Med., 368: 745-755. DOI: 10.1056/NEJMra1200894 